\title{
Análise factorial confirmatória da versão portuguesa do CSAI-2
}

Eduarda Maria Coelho, José Vasconcelos-Raposo, Hélder Miguel Fernandes

Centro de Investigação em Desporto, Saúde e Desenvolvimento Humano

da Universidade de Trás-os-Montes e Alto Douro (Portugal)

Coelho, E; Vasconcelos-Raposo, J.; Fernandes, H;

Análise factorial confirmatória da versão por-

tuguesa do CSAI-2. Motricidade 3(3): 73-82

\section{Resumo}

O objectivo deste estudo foi avaliar a estrutura factorial da versão portuguesa do CSAI-2, utilizando várias estruturas propostas em estudos anteriores, através da análise factorial confirmatória (AFC) em duas amostras distintas (uma de calibração e uma de validação). A amostra de calibração foi constituída por 287 alunos da licenciatura em Educação Física e Desporto e a amostra de validação foi constituída por 323 atletas de desportos individuais.

Os resultados da AFC realizada à estrutura original demonstraram uma inadequabilidade do modelo. As outras estruturas propostas por outros autores também foram analisadas e apresentaram índices de adequabilidade superiores, embora ainda não aceitáveis. O novo modelo que propomos, sem a escala de activação, revelou um ajustamento adequado para a amostra de calibração, sendo validada a sua adequabilidade pela segunda amostra $\left(\mathrm{X}^{2} / \mathrm{df}=2,645\right.$; $\mathrm{CFI}=0,916$; $\mathrm{GFI}=0,871$ e RMSEA=0,074). É sugerida a utilização deste novo modelo, em vez do original, na avaliação da ocorrência de pensamentos negativos, composto por duas escalas correlacionadas (negativismo e autoconfiança), e a continuação da verificação das propriedades psicométricas em investigações futuras.

Palavras-chave: negativismo, autoconfiança, CSAI-2 , análise factorial confirmatória.

\section{Abstract \\ Confirmatory factor analysis of the por- tuguese version of the CSAI-2}

The aim of this study was to assess the factorial structure of the Portuguese version of CSAI-2, using several structures suggested in previous studies, through confirmatory factor analysis (CFA) in two different samples (calibration and validation). The calibration sample consisted of 287 Physical Education and Sports students and the validation sample of 323 athletes of individual sports.

The results of CFA according to the original structure showed some inadequacy of the model. Other structures, suggested by different authors were analysed, revealing acceptable values levels of goodness of fit indices, though still not adequate ones. The new model that we suggest, without the arousal scale, has shown adequate adjustment for the calibration sample and its capability has been validated by the second sample $\left(X^{2} / d f=2,645 ; C F I=0,916 ; G F I=0,871\right.$ e RMSEA= 0,074). The use of the new model requires further validation, however, due to the obtained good of fitness scores its use in research is suggested. The new model consists of two negatively correlated scales (negativity and self-confidence). The subsequent confirmation of its psychometric properties is recommended for future studies.

Keywords: negativity, self-confidence, CSAI-2, confirmatory factor analysis. 


\section{Introdução}

Inicialmente, o conceito de ansiedade foi proposto para diagnosticar patologia mental ${ }^{30,31}$, sendo popularizado no contexto do desporto com o trabalho de Martens ${ }^{17}$, com o objectivo de avaliar e explicar o estado emocional que os atletas vivem nos momentos que antecedem as competições.

Vários autores contestam a forma como o termo ansiedade tem sido utilizado no contexto desportivo, referindo que a conotação negativa que o termo possui pode levar à compreensão errada que a ansiedade é sempre prejudicial para a performance ${ }^{12,14}$. Jones e Swain ${ }^{14}$ sugerem que quando a ansiedade é interpretada pelos atletas como benéfica para a performance, se chame a este estado emocional positivo de excitação, activação ou motivação.

Taylor ${ }^{24}$ considera mais apropriada a utilização do termo intensidade, em vez de ansiedade e activação. Este autor apresenta dois motivos que justificam esta opção: primeiro, porque o termo activação tem associado conotações sexuais, e o seu uso com atletas jovens, muitas vezes, produz uma reacção cómica que interfere com a compreensão da importância deste conceito na preparação para a competição desportiva; segundo, o termo ansiedade é tipicamente conhecido como tendo uma conotação negativa, como algo a ser evitado; no entanto, os atletas para terem uma prestação óptima necessitam de um determinado nível deste atributo. Taylor ${ }^{24}$ sugere que o termo intensidade não tem estas limitações e é visto pelos atletas como contribuindo para a performance desportiva óptima.

Recentemente, Vasconcelos-Raposo ${ }^{31}$ também propõe que se rejeite o conceito de ansiedade e que se proceda a uma reclassificação do estado emocional vivido pelos atletas no contexto desportivo, uma vez que está em contradição com a forma como este é utilizado pela psicologia clínica. Este autor considera inadequada a forma como a ansiedade é apresentada na psicologia do desporto, e que nunca foram apresentados os critérios que conduziram à sua utilização. Vasconcelos-Raposo ${ }^{31}$ afirma que não há dúvidas que existem atletas que sofrem de ansiedade, mas esses casos têm de ser diagnosticados de acordo com os critérios clínicos. Assim, e com base nos critérios do Diagnostic and Statistical Manual of Mental Disorders-III, este autor identifica os seguintes critérios que deverão estar presentes para diagnosticar alguém sofrendo de ansiedade: a disposição ansiosa deve ser contínua durante pelo menos um mês; os sintomas não devem estar associados a qualquer outra desordem mental e que o indivíduo tenha pelo menos dezoito anos de idade. O que está em causa é o uso deste conceito para classificar as sensações e dúvidas que os atletas podem ter relativamente às suas capacidades, nos momentos imediatamente anteriores à competição. Assim, Vasconcelos-Raposo ${ }^{31}$ recomenda que se proceda a estudos com o intuito de classificar correctamente as emoções no desporto. Este argumento foi apresentado pela primeira vez em $1988^{30}$, depois apresentado numa conferência em Zaragoza, em $1994^{30}$, e depois publicado em $2000^{31}$. No entanto, este apelo a estudos mais compreensivos vem desde a sua tese de doutoramento em 1993, em que o autor salienta que a linguagem que os atletas utilizam para descrever e classificar as suas emoções é claramente distinta da utilizada pelos psicólogos, concluindo que “...o conceito de ansiedade é algo que não encontra eco nas experiências dos atletas..." ${ }^{\prime 29}$.

Ao longo desta investigação, e tendo em conta as diferentes propostas dos autores, optaremos por utilizar o termo negativismo, por acharmos ser o que melhor se adequa ao estado emocional vivenciado pelos atletas antes da competição, principalmente, quando nos referimos à prevalência de pensamentos negativos, tal como tem sido definida a ansiedade cognitiva. Optamos 
pela utilização do termo activação em vez do de ansiedade somática, devido à similaridade entre as componentes fisiológicas de ambos.

No contexto desportivo foi desenvolvido o conceito de negativismo competitivo, que é uma alteração para uma situação específica de um constructo mais geral, adaptado especificamente para identificar disposições de traço de negativismo em situações competitivas ${ }^{17}$. O traço de negativismo competitivo pode ser definido como uma tendência para perceber as situações competitivas como ameaçadoras e responder com sentimentos de apreensão ou tensão ${ }^{17}$.

Ao mesmo tempo que surgem estes conceitos, diversos investigadores confirmaram que as medidas de negativismo, que são específicas para uma situação, predizem melhor o comportamento do que o fazem escalas gerais ${ }^{3}$. Em 1977, Martens sugere a necessidade de, também, criar instrumentos específicos para o desporto, com o objectivo de compreender melhor o comportamento do ser humano neste contexto, desenvolvendo o Sport Competition Anxiety Test (SCAT) e, mais tarde, o Competitive State Anxiety Inventory-1 (CSAI-1).

A partir de 1960 e mantém-se actualmente, os investigadores começaram a ter uma visão do negativismo como um constructo multidimensional, em vez de unidimensional, identificando diferentes componentes semelhantes aos conceitos de negativismo e activação ${ }^{19}$. Este avanço na conceptualização do negativismo contribuiu para uma melhor compreensão da sua relação com a performance ${ }^{13}$ e levou ao aparecimento de instrumentos de avaliação das suas componentes múltiplas. Martens, Burton e colaboradores ${ }^{18}$ conceptualizaram, construíram e validaram uma versão multidimensional do CSAI-1, a que chamaram CSAI-2. Este instrumento avalia a intensidade da activação, negativismo e autoconfiança.

O CSAI-2 é um dos questionários mais utilizados na verificação de alguns pressupostos teóricos inerentes à Teoria Multidimensional da Ansiedade, especificamente na verificação da relação existente entre as três escalas e o rendimento desportivo ${ }^{6,8,15,20,21,23,25}$. Nesta investigação, apesar de rejeitarmos o termo ansiedade proposto pela teoria original, aceitamos as dinâmicas sugeridas pelos autores entre as várias dimensões e o rendimento desportivo. A Teoria Multidimensional propõe que as três dimensões se relacionam de forma diferente com o rendimento: o negativismo de forma linear e negativa; a activação relaciona-se de forma curvilínea (em U-invertido) e a autoconfiança tem uma relação linear e positiva com o rendimento.

Burton $^{3}$ refere que, apesar de existir independência conceptual entre o negativismo e a activação, há uma relação recíproca entre estas duas componentes, embora seja difícil especificar a contribuição exacta de cada na situação competitiva real. Diversos estudos comprovam que as duas componentes do negativismo se relacionam positivamente ${ }^{5,7,7,10,11,16,21,22,28,33,34}$. E que, a autoconfiança se relaciona negativamente tanto com a activação ${ }^{7,10,15,1,21}$, como com o negativismo ${ }^{7,9101,16,21}$.

Martens, Burton e colaboradores ${ }^{18}$ realizaram quatro estudos com o objectivo de testar a validade da forma final do CSAI-2, demonstrando os resultados que este questionário possui validade de constructo e verificaram o seu uso no cálculo do negativismo, activação e autoconfiança no desporto. Desde esse momento, que o CSAI-2 tem sido visto como um dos instrumentos mais fidedignos e válidos para a avaliação do negativismo no contexto desportivo ${ }^{3}$.

Estudos iniciais que avaliaram a homogeneidade dos itens dentro das escalas demonstraram que o CSAI-2 tem forte consistência interna nas suas três escalas, com coeficientes de alpha de Cronbach entre 0,79 e 0,9018. Recentemente, diversas investigações realizadas também comprovam a elevada consistência interna das escalas do CSAI-2,101,16,26,27. 
No que diz respeito à validade factorial do CSAI-2, diversos estudos recentes não comprovam a estrutura original ${ }^{7,16}$, sendo três destas investigações acerca da tradução e adaptação do questionário para Grego ${ }^{10,27,28}$.

Em 1998, Tsorbatzoudis e colaboradores ${ }^{27}$ realizaram um estudo com atletas gregos de desportos colectivos, com o objectivo de verificar a validade factorial do CSAI-2, utilizando a análise factorial exploratória. Os resultados confirmaram a estrutura inicialmente proposta por Martens, Burton e colaboradores ${ }^{18} \mathrm{com}$ excepção de três itens $(4,13,25)$, que carregavam negativamente na autoconfiança, em vez de no negativismo.

Lane e colaboradores ${ }^{16}$ realizaram um estudo com o mesmo objectivo do anterior, mas utilizando a análise factorial confirmatória (AFC) em duas amostras. As medidas de adequabilidade obtidas foram baixas em ambas as amostras, sugerindo que a estrutura factorial original é imperfeita. Os autores indicam que a limitação da escala de negativismo deriva da utilização dos termos concerned em vez de worried, sugerindo que estar concerned acerca da performance não significa que o atleta vivência pensamentos negativos, mas sim que este reconhece a importância e dificuldade do desafio e está a preparar-se para o enfrentar.

Posteriormente, Iosifidou e Doganis ${ }^{10}$ utili- $^{-}$ zaram a AFC para verificar a validade factorial do CSAI-2, com uma amostra de nadadores avaliada em dois momentos distintos (um dia antes e uma hora antes da competição). Os resultados demonstraram índices de adequabilidade superiores para os dados recolhidos uma hora antes da competição, no entanto os valores obtidos não confirmaram a estrutura original. Os autores atribuem os baixos valores dos índices de adequabilidade aos possíveis carregamentos cruzados existentes no modelo.

Recentemente, os resultados da AFC de um estudo de Tsorbatzoudis e colaboradores ${ }^{28}$ também não confirmaram a estrutura de três factores proposta por Martens, Burton e colaboradores ${ }^{18}$ e a estrutura de dois factores correlacionados (negativismo e activação), seguindo a sugestão de Lane et al. ${ }^{16}$, que era a presença da autoconfiança que provocava a ineficácia do modelo. A exclusão do item 25 do factor cognitivo, tal como sugerido por Tsorbatzoudis e colaboradores $^{27}$, resultou em medidas de adequabilidade aceitáveis, sugerindo que o CSAI-2 pode ser usado para avaliar a activação com nove itens e o negativismo com oito, mantendo as duas escalas correlacionadas.

Finalmente, Cox e colaboradores ${ }^{7}$ realizaram um estudo com o objectivo de rever a estrutura factorial do CSAI-2, também através da AFC e usando duas amostras, uma de calibração e uma de validação. Os resultados da amostra de calibração demonstraram baixos índices de adequabilidade da estrutura original, sendo eliminados 10 itens que carregavam em mais de um factor, ficando a estrutura proposta composta por 17 itens. Os resultados da AFC da nova estrutura proposta pelos autores, revelou bons índices de adequabilidade dos dados ao modelo.

As investigações anteriormente descritas sugerem a necessidade de realizar mais estudos para a verificação da estrutura factorial do CSAI-2, e, especialmente, da escala traduzida e validada para Português. De facto, a existência de uma escala válida para a avaliação da ocorrência de pensamentos negativos fornece uma melhor compreensão dos estados de negativismo no contexto do desporto. Definimos como objectivo deste estudo avaliar a estrutura factorial da versão Portuguesa do CSAI-2, utilizando várias estruturas propostas pelos autores nos estudos descritos anteriormente, usando a AFC em duas amostras distintas. Primeiro, pretendeu-se avaliar a estrutura factorial do questionário, segundo os vários modelos propostos, na amostra de calibração e fazer as alterações necessárias; posteriormente, a estrutura resultante foi testada na amostra de validação. 


\section{Metodologia}

\section{Amostra}

A amostra de calibração foi constituída por 287 alunos da licenciatura em Educação Física e Desporto com mais de um ano de prática desportiva, de ambos os sexos (187 alunos masculinos e 100 femininos), praticantes de modalidades individuais e colectivas e de diversos níveis competitivos. Todos os elementos da amostra tinham idades superiores a 18 anos $(20,64 \pm 2,17)$, e apresentavam em média cerca de 7 anos de prática desportiva $(6,63 \pm 3,78)$.

A amostra de validação foi composta por 323 atletas praticantes de seis modalidades desportivas individuais (atletismo, natação, ginástica, judo, luta livre e badminton), de ambos os sexos (207 atletas masculinos e 116 femininos) e com nível competitivo mínimo de Nacional. Todos os membros da amostra tinham idades superiores a 16 anos $(20,48 \pm 4,02)$, e cerca de 10 anos de prática desportiva $(10,13 \pm 4,96)$.

\section{Procedimentos}

Os questionários referentes à amostra de calibração, composta pelos estudantes de Educação Física e Desporto, foram recolhidos antes do início de uma aula, tendo sido solicitado previamente autorização para o efeito ao professor da referida disciplina. Os dados respeitantes à amostra de validação, constituída por atletas, foi recolhida em situação de estágio da selecção nacional ou treino nos respectivos clubes, após dada autorização dos Presidentes e Técnicos Nacionais das respectivas Federações.

O Inventário de Estado de Ansiedade Competitiva-2 (Competitive State Anxiety Inventory-2, CSAI-2) foi desenvolvido por Martens, Burton e colaboradores $^{18}$ para avaliar a intensidade do negativismo, da activação e da autoconfiança em situações competitivas, sendo validado para a população portuguesa por Vasconcelos-Raposo ${ }^{32}$.
O CSAI-2 é constituído por 27 itens, com respostas classificadas numa escala de Likert de 4 pontos, entre 1 (nada) e 4 (muito). Cada uma das três escalas consiste em nove itens, sendo o valor de cada escala calculado através da soma dos itens. Os valores de cada escala variam entre um mínimo de 9 e um máximo de 36.

\section{Estatística}

Para o tratamento estatístico dos dados foram utilizados os programas SPSS 13.0 e o AMOS 6.0 (Analysis of Moment Structures). Verificou-se as propriedades psicométricas do CSAI-2, através do cálculo do alpha de Cronbach e da AFC.

Com base nas várias estruturas factoriais propostas nos estudos anteriormente descritos, foi realizada a AFC com a amostra de alunos e atletas. Em todos os modelos testados foi utilizado o método de estimação maximum likelihood. As medidas de avaliação do ajustamento utilizadas para verificar a adequabilidade do modelo aos dados foram as seguintes: ratio chi square statistics/degrees of freedom $\left(\mathrm{X}^{2} / d f\right)$, comparative fit index (CFI), goodness of fit index (GFI), root mean square error of approximation (RMSEA) e Akaike information criterion (AIC).

Inicialmente, o qui-quadrado foi a estatística mais utilizada para avaliar a adaptabilidade do modelo, indicando um qui-quadrado significativo que o modelo em estudo não se adapta aos dados. Posteriormente, foi reconhecida a elevada sensibilidade deste teste ao tamanho da amostra ${ }^{2}$, sendo sugerido a utilização do rácio $\mathrm{X}^{2} / \mathrm{df}$. $\mathrm{O}$ rácio do $\mathrm{X}^{2} / \mathrm{df}$ tem sido usado como índice de ajustamento do modelo, no entanto, não há consenso acerca do valor que se considera um ajustamento adequado, sendo sugerido valores inferiores a $3^{1}$. O CFI avalia a adequabilidade do modelo em relação ao modelo independente, variando os valores entre 0 e 1 , com valores superiores a 0,90 indicando um ajustamento adequado $^{4}$. O GFI mede a quantidade relativa 
de variância e covariância conjuntamente explicadas pelo modelo, varia entre 0 e 1 , com valores superiores a 0,9 indicando um ajustamento adequado $^{4}$. O RMSEA analisa a discrepância no ajustamento entre as matrizes estimadas e as observadas, varia entre 0 e 1 , com valores entre 0,08 e 0,1 indicando um modelo medíocre e superiores a 0,1 uma adequabilidade pobre ${ }^{4}$. Na comparação de dois ou mais modelos usamos o AIC, demonstrando os valores mais baixos uma maior adequabilidade do modelo ${ }^{4}$.

\section{Resultados}

\section{Consistência Interna}

A consistência interna foi boa para as três escalas do CSAI-2, em ambas as amostras. Para a amostra dos alunos os valores do alpha de Cronbach foram de 0,84 para o negativismo, 0,88 para a activação e para a autoconfiança de 0,91 . Para a amostra de atletas os valores obtidos foram de 0,85 para o negativismo, 0,88 para a activação e 0,90 para a autoconfiança.

\section{Análise Factorial Confirmatória}

O quadro 1 apresenta os valores das medidas do ajustamento dos modelos propostos pelos autores referidos anteriormente, relativos à amostra de calibração desta investigação.
Como se verifica no quadro 1 , a avaliação do modelo original do CSAI-2 sugerido por Martens, Burton e colaboradores ${ }^{18}$, que assume a existência de três factores correlacionados, demonstra uma inadequabilidade do modelo, tendo todas as medidas de ajustamento valores inaceitáveis $\left(\mathrm{X}^{2} / \mathrm{df}=3,242, \mathrm{CFI}=0,828\right.$, GFI $=$ $0,780$ e RMSEA $=0,089)$. Foram obtidos valores das medidas de ajustamento idênticos para a amostra de atletas (ver quadro 2).

O modelo seguinte, que resulta da eliminação da escala de autoconfiança surge da sugestão de Lane e colaboradores ${ }^{16}$ que a ineficácia do modelo inicial pode ser devido à presença da autoconfiança, demonstrou uma melhor adequação à amostra que o anterior. Os índices de adequabilidade são superiores mas não aceitáveis $\left(\mathrm{X}^{2} / \mathrm{df}=3,098, \mathrm{CFI}=0,862, \mathrm{GFI}=0,873, \mathrm{e}\right.$ RMSEA $=0,086)$. Os resultados foram semelhantes na amostra de atletas (ver quadro 2).

O $3 .^{\circ}$ modelo testado, resulta das analises realizadas por Tsorbatzoudis e colaboradores ${ }^{27,28}$ que sugerem a eliminação do item 25 para a melhoria dos índices de adequabilidade do modelo, devido a carregar fortemente na autoconfiança, embora tenha sido desenvolvido para reflectir a prevalência de pensamentos negativos. Comparativamente ao modelo anterior, $\mathrm{o} \mathrm{X}^{2} / \mathrm{df}$ é mais elevado e os índices de adequabilidade apresentam valores inferiores, o que significa que o modelo proposto que não se adequa à amostra

Quadro 1: Valores das medidas de ajustamento para a amostra de calibração (alunos).

\begin{tabular}{|c|c|c|c|c|c|}
\hline & Original $^{18}$ & $\begin{array}{c}\text { Negativismo } \\
\text { e } \\
\text { activação }^{16}\end{array}$ & $\begin{array}{l}\text { Negativismo } \\
\text { e activação } \\
\text { sem item } 25^{27,28}\end{array}$ & $\begin{array}{c}\text { Negativismo } \\
\text { e } \\
\text { autoconfiança }\end{array}$ & $\begin{array}{c}\text { Modelo } \\
\text { final } \\
\text { proposto }\end{array}$ \\
\hline$X^{2}$ & 1040,659 & 415,125 & 391,640 & 395,275 & 286,048 \\
\hline df & 321 & 134 & 118 & 134 & 130 \\
\hline$p$ & 0,000 & 0,000 & 0,000 & 0,000 & 0,000 \\
\hline $\mathrm{X}^{2} / \mathrm{df}$ & 3,242 & 3,098 & 3,319 & 2,950 & 2,200 \\
\hline CFI & 0,828 & 0,862 & 0,869 & 0,894 & 0,937 \\
\hline GFI & 0,780 & 0,873 & 0,864 & 0,854 & 0,895 \\
\hline RMSEA & 0,089 & 0,086 & 0,090 & 0,083 & 0,065 \\
\hline AIC & 1154,659 & 489,125 & 461,640 & 469,275 & 368,048 \\
\hline
\end{tabular}


Quadro 2: Valores das medidas de ajustamento para a amostra de validação (atletas).

\begin{tabular}{lcccc} 
& Original ${ }^{18}$ & $\begin{array}{c}\text { Negativismo } \\
\mathbf{e}\end{array}$ & $\begin{array}{c}\text { Negativismo } \\
\text { e activação } \\
\text { sem item 25 27,28 }\end{array}$ & $\begin{array}{c}\text { Modelo } \\
\text { final } \\
\text { proposto }\end{array}$ \\
\hline $\mathrm{X}^{2}$ & 1020,140 & 366,998 & 338,953 & 343,804 \\
$\mathrm{df}$ & 321 & 134 & 118 & 130 \\
$\mathrm{p}$ & 0,000 & 0,000 & 0,000 & 0,000 \\
$\mathrm{X}^{2} / \mathrm{df}$ & 3,178 & 2,739 & 2,872 & 2,645 \\
$\mathrm{CFI}$ & 0,835 & 0,898 & 0,897 & 0,916 \\
$\mathrm{GFI}$ & 0,770 & 0,877 & 0,880 & 0,871 \\
$\mathrm{RMSEA}$ & 0,085 & 0,076 & 0,078 & 0,074 \\
AIC & 1134,140 & 440,998 & 408,953 & 425,804 \\
\hline
\end{tabular}

Figura 1: Modelo final proposto.

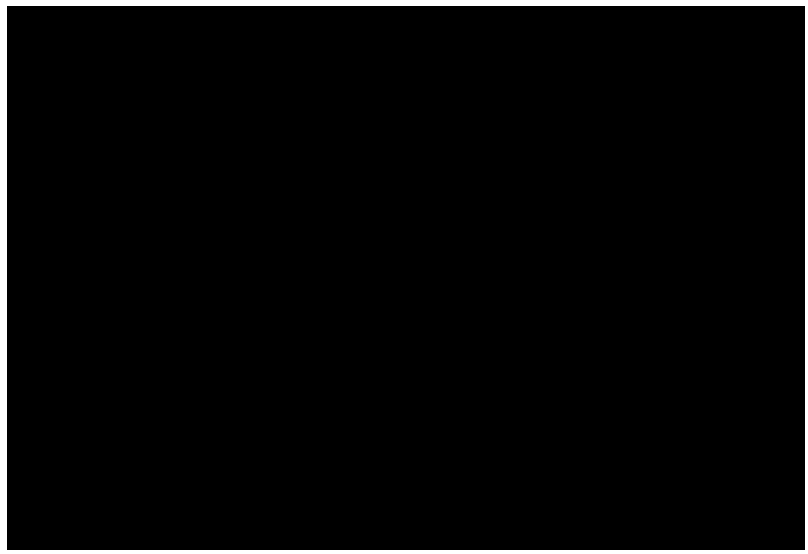

de estudantes $\left(\mathrm{X}^{2} / \mathrm{df}=3,319, \mathrm{CFI}=0,869\right.$, GFI= $=0,864$, e RMSEA $=0,090)$. Relativamente à amostra de atletas, os índices de adequabilidade deste modelo são um pouco superiores, não se considerando ainda aceitáveis (ver quadro 2).

O modelo que propomos nesta investigação inclui unicamente as escalas de negativismo e autoconfiança, verificando-se um aumento dos valores das medidas de ajustamento para valores próximo do considerado aceitável $\left(\mathrm{X}^{2} / \mathrm{df}=2,950\right.$, $\mathrm{CFI}=0,894$, GFI $=0,854$, e RMSEA $=0,083$ ). Através da analise dos índices de modificação (output do AMOS) e da ligação entre os erros dentro da mesma escala, chegamos a uma estrutura final semelhante à original, apenas se acrescentando as ligações entre os erros dos itens 3-21, 3-6, 15-18 e 18-27 (ver figura 1), aumen- tando os valores das medidas de ajustamento para valores considerados bons $\left(\mathrm{X}^{2} / \mathrm{df}=2,200\right.$, $\mathrm{CFI}=0,937, \mathrm{GFI}=0,895$ e RMSEA $=0,065)$.

Simultaneamente, quando comparamos os cinco modelos entre si usando o valor de AIC, verificamos que o modelo original de Martens, Burton e colaboradores ${ }^{18}$ é o que apresenta valores mais elevados $(\mathrm{AIC}=1154,659)$ e o modelo final proposto é o que apresenta valores mais baixos $(\mathrm{AIC}=368,048)$, o que significa uma melhor adequabilidade à amostra do modelo composto pelas escalas de negativismo e autoconfiança.

Com o objectivo de validar o modelo anteriormente proposto, realizamos a AFC com uma amostra de atletas, sendo os resultados apresentados na última coluna do quadro 2 . 
O novo modelo proposto, os coeficientes path estandardizados e as correlações entre factores da amostra de validação são apresentados na figura 1. Todos os coeficientes path dos factores (variáveis latentes) para as variáveis avaliadas são próximos ou superiores a 0,50 e significativos (unicamente o item 19 carrega a 0,48 no negativismo), com valores de $\mathrm{p}<0,001$. Conjuntamente, os valores das medidas de ajustamento obtidas pela AFC na amostra de atletas validam a adequabilidade do novo modelo proposto $\left(\mathrm{X}^{2} / \mathrm{df}=2,645, \mathrm{CFI}=0,916, \mathrm{GFI}=0,871\right.$ e RMSEA = 0,074).

\section{Discussão}

O objectivo do presente estudo foi avaliar a estrutura factorial da versão Portuguesa do CSAI-2. Este instrumento era considerando uma ferramenta válida e fidedigna para a avaliação do negativismo, activação e autoconfiança $a^{27,28}$, sendo, no entanto, recentemente questionada em diversas investigações as suas propriedades psicométricas, no que diz respeito à validade factorial ${ }^{3}$. As conclusões deste estudo apoiam os autores referidos anteriormente, no que diz respeito à falta de validade factorial da escala original do CSAI-2 $2^{7,1016,27,28}$, sendo sugerido um modelo composto apenas pelas escalas de negativismo e autoconfiança.

No entanto, se em relação à estrutura factorial, as medidas de adequabilidade obtidas demonstram valores inaceitáveis para a estrutura original ${ }^{18}$; no que diz respeito à consistência interna das escalas do CSAI-2, os resultados confirmam a existência de valores de Alpha de Cronbach elevados (variam entre 0,84 e 0,91 ). Os valores de alpha obtidos para as três escalas, em ambas as amostras, estão de acordo com outras investigações realizadas ${ }^{18}$.

O modelo que propomos, sem a escala de activação, revela medidas de ajustamento mais adequadas comparativamente aos outros três modelos analisados, rejeitando a justificação dada por estes autores que o problema do questionário está relacionado com a escala de autoconfiança, e à forma como esta escala foi desenvolvida (surgiu da analise factorial exploratória realizada e sem suporte teórico).

No novo modelo que propomos são utilizadas estratégias para a melhoria dos índices de adequabilidade que provêm da Teoria Multidimensional da Ansiedade. A eliminação da escala de activação deriva desta ser bastante afectada pelo erro do tipo desejabilidade social (o indivíduo distorcer as suas respostas de uma forma socialmente desejável ${ }^{18}$; e pelo estado de activação se correlacionar menos com a performance do que o negativismo, devido a este estado se dissipar no início da competição desportiva ${ }^{18}$.

A existência de uma correlação negativa entre as duas escalas $(r=-0,79)$ também reforça a validade do nosso modelo, de facto nos estudos originais de desenvolvimento deste questionário, a média das correlações testadas em várias amostras é de $-0,61^{18}$. Outros estudos realizados também comprovam a relação negativa existentes entre as duas escalas?.

A utilização de duas amostras de atletas com características distintas nesta investigação, além de nos permitir validar a estrutura factorial que propomos, fornece um excelente suporte para o seu uso com diferentes populações de atleta. No entanto, aconselhamos a continuação da verificação das propriedades psicométricas do modelo proposto sempre que for utilizado o questionário.

Das várias análises realizadas pode-se concluir que é preferível a utilização de uma escala de dois factores a uma de três, como foi originalmente proposto por Martens, Burton e colaboradores ${ }^{18}$ para a avaliação do negativismo no contexto desportivo. Os resultados desta investigação sugerem uma adaptação do CSAI-2 a que chamaremos Escala de Negativismo e Autoconfiança (ENAC), com as escalas de negativismo e autoconfiança correlacionadas. 


\section{Correspondência}

José Vasconcelos-Raposo

Rua Dr. Manuel Cardona

5000-558 Vila Real, Portugal

E-mail: j.vasconcelos.raposo@gmail.com

\section{Referências}

1. Aroian, K. e Norris, A. (2005). Confirmatory factor analysis. In B. Munro (Ed.), Statistical methods for health care research ( $5^{\mathrm{a}}$ ed., pp. 351-375): Lippincott Williams \& Wilkins.

2. Bryant, F. e Yarnold, P. (2004). Principalcomponents analysis and exploratory and confirmatory factor analysis. In L. Grimm \& P. Yarnold (Eds.), Reading and understanding multivariate statistics (pp. 99-136). Washington: American Psychological Association.

3. Burton, D. (1998). Measuring competitive state anxiety. In J. Duda (Ed.), Advances in sport and exercise psychology measurement (pp. 129-148). Morgantown: Fitness Information Technology.

4. Byrne, B. (2001). Structural equation modeling with AMOS: Basic concepts, applications and programming. New Jersey: Lawrence Erlbaum Associates.

5. Cerin, E. (2004). Predictors of competitive anxiety direction in male tae kwon do practitioners: a multilevel idiographic/nomothetic interactional approach. Psychology of Sport \& Exercise, 5, 497-516.

6. Chapman, C.; Lane, A.; Brierley, J. e Terry, P. (1997). Anxiety, self-confidence and performance in tae kwon-do. Perceptual and Motor Skills, 85, 1275-1278.

7. Cox, R.; Martens, M. e Russell, W. (2003). Measuring anxiety in athletics: The revised Competitive State Anxiety Inventory-2. Journal of Sport \& Exercise Psychology, 25, 519-533.

8. Craft, L.; Magyar, M.; Becker, B. e Feltz, D. (2003). The relationship between the Competitive State Anxiety Inventory- 2 and sport performance: A meta-analysis. Journal of Sport \& Exercise Psychology, 25, 44-65.
9. Edwards, T. e Hardy, L. (1996). The interactive effects of intensity and direction of cognitive and somatic anxiety and self-confidence upon performance. Journal of Sport \& Exercise Psychology, 18, 296-312.

10. Iosifidou, P. e Doganis, G. (2001). Confirmatory factor analysis of the Greek version of the Competitive State Anxiety Inventory-2. International Journal of Sport Psychology, 32, 400-405.

11. Jones, G. e Hanton, S. (1996). Interpretation of competitive anxiety symptoms and goal attainment expectancies. Journal of Sport \& Exercise Psychology, 18, 144-157.

12. Jones, G. e Hanton, S. (2001). Pre-competitive feeling states and directional anxiety interpretations. Journal of Sports Sciences, 19, 385-395.

13. Jones, G. e Hardy, L. (1990). The academic study of stress in sport. In J. Jones e L. Hardy (Eds.), Stress and performance in sport (pp. 3-16). New York: John Wiley \& Sons.

14. Jones, G. e Swain, A. (1992). Intensity and direction as dimensions of competitive state anxiety and relationships with competitiveness. Perceptual and Motor Skills, 74, 467-472.

15. Jones, G., Swain, A., \& Hardy, L. (1993). Intensity and direction dimensions of competitive state anxiety and relationships with performance. Journal of Sports Sciences, 11, 525-532.

16. Lane, A.; Sewell, D.; Terry, P; Bartram, D. e Nesti, M. (1999). Confirmatory factor analysis of the Competitive State Anxiety Inventory-2. Journal of Sports Sciences, 17, 505-512.

17. Martens, R. (1977). Sport competition anxiety test. Champaign: Human Kinetics.

18. Martens, R.; Burton, D.; Vealey, R.; Bump, L. e Smith, D. (1990). Development and validation of the Competitive State Anxiety Inventory-2 (CSAI-2). In R. Martens, R. Vealey e D. Burton (Eds.), Competitive anxiety in sport (pp. 117-213). Champaign: Human Kinetics.

19. Martens, R.; Vealey, R. e Burton, D. (1990). Competitive anxiety in sport. Champaign: Human Kinetics. 
20. Martin, J. e Gill, D. (1991). The relationships among competitive orientation, sport-confidence, self-efficacy, anxiety, and performance. Journal of Sport \& Exercise Psychology, 13, 149-159.

21. Maynard, I.; Smith, M. e Warwick-Evans, L. (1995). The effects of a cognitive intervention strategy on competitive state anxiety and performance in semiprofessional soccer players. Journal of Sport \& Exercise Psychology, 17, 428-446.

22. Muchuane, D. e Duarte, A. (2003). Ansiedade pré-competitiva de atletas moçambicanos praticantes de natação e atletismo. In A. Prista, A. Marques, A. Madeira e S. Saranga (Eds.), Actividade física e desporto: Fundamentos e contextos (pp. 260-269). Porto: FCDEF-UPM, FCDEF-UP, Faculdade de Medicina Universidade Eduardo Mondlane.

23. Rodrigues, R. e Cruz, J. (1997). Autoconfiança, ansiedade e rendimento na natação de alta competição: Estudo com os atletas de elite nacional. Psicologia: Teoria, investigação e prática, 3, 491-521.

24. Taylor, J. (1996). Intensity regulation and athletic performance. In J. Raalte e B. Brewer (Eds.), Exploring sport and exercise psychology (pp. 75-106). Washington: American Psychological Association.

25. Terry, P.; Mayer, J. e Howe, B. (1998). Effectiveness of a mental training program for novice scuba divers. Journal of Applied Sport Psychology, 10, 251-267.

26. Thomas, O.; Maynard, I. e Hanton, S. (2004). Temporal aspects of competitive anxiety and self-confidence as a function of anxiety perceptions. The Sport Psychologist, 18, 172-187.
27. Tsorbatzoudis, H.; Barkoukis, V.; Rodafinos, A. e Grouios, G. (1998). A test of the reliability and factorial validity of the Greek version of the CSAI-2. Research Quarterly for Exercise and Sport, 69, 416-419.

28. Tsorbatzoudis, H.; Barkoukis, V.; Sideridis, G. eGrouios, G. (2002). Confirmatory factor analysis of the Greek version of the Competitive State Anxiety Inventory (CSAI-2). International Journal of Sport Psychology, 33, 182-194.

29. Vasconcelos-Raposo, J. (1993). Os factores psico-sócio-culturais que influenciam e determinam a busca da excelência pelos atletas de elite desportiva portuguesa. Unpublished Tese de Doutoramento, Universidade de Trás-os-Montes e Alto Douro, Vila Real.

30. Vasconcelos-Raposo, J. (1994). Explorando as limitações do conceito de ansiedade no desporto. Paper presented at the XIV Congreso de la Asociacion Aragoneza de Tecnicos de Natacion, Zaragosa.

31. Vasconcelos-Raposo, J. (2000). Explorando as limitações do conceito de ansiedade no desporto. ALEU: Revista de Desporto da UTAD, 2, 47-66.

32. Vasconcelos-Raposo, J. (Ed.). (1995). Diferenças por classificação no nivel de ansiedade cognitiva, somática e auto-confiança: UTAD.

33. Vasconcelos-Raposo, J. e Lazaro, J. (2000). Niveis de ansiedade cognitiva, somática e auto-confiança de jovens saltadores e lançadores portugueses. Paper presented at the II Congresso Luso-Espanhol de Psicologia do Desporto e do Exercício, Évora.

34. Wiggins, M. (1998). Anxiety intensity and direction: Preperformance temporal patterns and expectations in athletes. Journal of Applied Sport Psychology, 10, 201-211. 